\title{
Estado del arte sobre talleres de escritura filosófica
}

\author{
— THE STATE-OF-THE-ART OF WORKSHOPS ON PHILOSOPHICAL WRITING \\ ESTADO DA ARTE SOBRE OFICINAS DE ESCRITA FILOSÓFICA
}

\author{
Ana Isabel Durán Vélez* / anai.duran@gmail.com
}

\section{Resumen}

Este estado del arte presenta corrientes dominantes en la realización de talleres de escritura filosófica. El criterio utilizado para clasificar los talleres es el lugar que la escritura tiene en dicha práctica y se han identificado tres grandes corrientes. La primera corriente enseña habilidades de redacción y de argumentación a los participantes para poder presentar ensayos y textos académicos destinados a la publicación y a la evaluación. La segunda, comprende los talleres que al salir del ámbito académico buscan un conocimiento de sí mismo a partir del diálogo con la filosofía y con otros. Finalmente, en la tercera corriente se encuentran los talleres en los que se busca generar las habilidades para razonar correctamente.

\section{Abstract}

This state-of-the-art presents the main trends in organizing workshops on philosophical writing. The criterion used to classify these workshops is the role of writing in them; three main trends were identified. The first one teaches participants how to write clearly and how to present arguments coherently in order to produce essays and articles to be published and evaluated. The second type consists of workshops that, outside of the academic environment, are oriented toward self-knowledge through dialogues with philosophy and other disciplines. Finally, the third type of workshops are those that seek to create in students the abilities needed to reason correctly.

\section{Resumo}

Este Estado da Arte apresenta correntes dominantes na realização de oficinas de escrita filosófica. O critério utilizado para classificar as oficinas é o lugar que a escrita tem nessa prática. Três grandes correntes foram identificadas: a primeira corrente ensina habilidades de redação e de argumentação aos participantes para poder apresentar ensaios e textos acadêmicos destinados à publicação e à avaliação; a segunda, compreende as oficinas que, ao sair do âmbito acadêmico, procuram um conhecimento de sim mesmo a partir do diálogo com a filosofia e com outros. Finalmente, na terceira corrente, podemos encontrar as oficinas nas que se procura gerar as habilidades para razoar corretamente.

\section{Palabras clave}

Filosofía, talleres de escritura filosófica, escritura, lectura, creatividad

\section{Keywords}

Philosophy, workshops on philosophical writing, writing, reading, creativity.

\section{Palavras chave}

Filosofia, oficinas de escrita filosófica, escrita, leitura, criatividade.

\footnotetext{
1 Docente de escritura y lectura en el grupo LEA, filósofa de la Universidad Javeriana y mágister en filosofía de la Universidad de Poitiers, Francia.
}

Fecha de recepción: 14 de enero de 2016 / Fecha de aprobación: 13 de abril de 2016 
El objetivo del presente escrito es mostrar las líneas de desarrollo de los talleres de escritura filosófica que se realizan como prácticas pedagógicas, bien sea fuera o dentro del aula de clase. Está enmarcado dentro del proyecto de investigación Filosofía, escritura y vida de la Universidad Pedagógica Nacional1 ${ }^{1}$, en el que se busca indagar cuál es el papel de la escritura en el marco de una noción de filosofía como modo de vida. En la medida en que el diseño de experiencias de escritura se nutre de los ejemplos de otras personas y por los caminos que ya se han construido, el presente trabajo muestra un mapa de los diferentes tipos de ejercicios pedagógicos que buscan Ilegar a la escritura filosófica.

De este modo, se han rastreado diferentes metodologías que se han agrupado según el rol que la escritura juega en el proceso pedagógico. Esto ha permitido clasificar los talleres de escritura filosófica en tres grandes clases: a) aquellos que buscan fomentar hábitos de escritura que den lugar a un texto claro y argumentado en el ámbito académico, pues su fin es que los textos sean evaluados o publicados. b) aquellos talleres que parten del debate y de la reflexión filosófica, con el fin de generar formas de escritura personales que den lugar a la apropiación de sí mismo y del mundo, es decir, talleres que no tienen como fin ni la evaluación ni la publicación. c) por último, encontramos talleres que utilizan diversas estrategias para desarrollar habilidades de razonamiento abstracto, la creatividad y el pensamiento lógico en contextos escolares como preparación para enfrentar los desafíos en la universidad.

Nuestro interés no consiste en juzgar si las nociones de filosofía que se manejan son adecuadas o correctas, sino entender qué nociones de filosofía dan lugar a qué tipo de prácticas y qué papel juega la escritura en el desarrollo de estas prácticas. De este modo, no nos comprometemos con una noción específica de filosofía, sino con la posibilidad de definir los conceptos en las prácticas. También nos interesa examinar las prácticas mismas con el fin de comprender el modo como los diferentes autores y profesores enfocan sus actividades hacia el desarrollo de una competencia o de una habilidad.

1 Proyecto financiado por el Centro de Investigaciones de la Universidad Pedagógica Nacional, código DCS 407-15. Hace parte de los desarrollos del grupo Filosofía y Enseñanza de la Filosofía.
Para desarrollar la investigación, se hizo una búsqueda del problema que nos atañe en libros, revistas, páginas web y eventos que dieran cuenta de la realización de talleres de escritura filosófica o que mostraran estrategias pedagógicas para el desarrollo de la escritura y del pensamiento en filosofía. En términos de las revistas, se revisaron: Universitas philosophica de la Universidad Javeriana, Ideas y valores de la Universidad Nacional y Teaching philosophy. Además, se revisaron las bases de datos de la Universidad Pedagógica y de las universidades ya mencionadas. Para acceder a los debates contemporáneos sobre escritura se utilizó la información del Grupo francés de la nueva educación GFEN, la página web de Michel Tozzi y del Instituto de prácticas filosóficas de París. Si bien la bibliografía que se presenta acá no da cuenta de la totalidad de talleres de escritura filosófica, sí se examinan casos que ilustran cada una de las categorías mencionadas.

Teniendo en cuenta lo anterior, el presente texto busca mostrar los talleres de escritura y examinar el papel que esta última juega en el desarrollo de un proceso pedagógico. Para ello, tenemos las tres categorías ya mencionadas, las cuales dan lugar a los tres momentos que componen este estado del arte. El primer momento responde a la categoría de los talleres de argumentación. El segundo, presenta los talleres de apropiación de sí mismo y del mundo a partir de la escritura. En el tercero, contamos con los talleres que buscan generar competencias y habilidades como el razonamiento lógico, el discernimiento y la creatividad. Con todo, pretendemos rastrear y agrupar las distintas experiencias pedagógicas que se ejecutan en torno al fomento de la escritura en relación con la formación filosófica. Al agruparlas por categorías se identifican tendencias y líneas de acción, a la vez que se vislumbran posibles estrategias por explorar. En este sentido, la aproximación que ofrecemos aquí es una herramienta tanto para investigadores en este campo, como para quienes se interesan por implementar propuestas de escritura filosófica.

\section{La escritura como exposición clara y argumentada de una idea}

Comenzamos con los talleres que involucran la escritura académica como los ensayos, los artículos y la disertación en Francia. Estos talleres despliegan dife- 
rentes metodologías que buscan en el estudiante una comprensión y desarrollo claro de un tema filosófico. Aunque, como en el caso de Solomon (2001), el punto de partida sean preguntas íntimas o lecturas redactadas en un lenguaje cotidiano, el objetivo pedagógico tras este tipo de escritura consiste en realizar una exposición argumentada, claramente articulada, y bien escrita de un tema filosófico. Esto puede lograrse, bien sea a partir de talleres que clarifiquen que debe hacerse un ensayo o artículo, o a partir de ciertas actividades que den lugar a una mejor escritura. Demos entonces paso a la descripción de los talleres.

\section{Talleres de escritura de géneros académicos}

El manual de escritura filosófica de la Universidad de Harvard (Chudnoff, 2007) parte de una noción de filosofía como la capacidad de "presentar la defensa de una tesis" (p. 5). De este modo, una reflexión sobre la audiencia es importante para saber exactamente qué y cómo se debe escribir. Si queremos defender una tesis, es importante saber sobre los tipos de argumentos, sobre lógica y sobre la motivación de la argumentación. Así, es posible no solo expresar una tesis, sino también justificar la validez de un argumento que no se había pensado antes. Si bien los argumentos son necesarios, hay que escribir con una cierta narrativa y estilo. La forma en este caso permite organizar los argumentos y presentarlos de tal modo que se sigan y que guarden una coherencia interna que sea agradable al lector.

En la misma línea se encuentran los talleres de Joe Cruz (2005) en la Universidad de Williams. Para él, la escritura es filosófica en cuanto trata de manera crítica y organizada textos propios de la historia de la filosofía. Así, en su página encontramos un ejercicio muy útil a la hora de escribir, pues trae pautas para desarrollar la introducción, los borradores y el texto final. Además, el autor muestra un texto escrito y lo comenta de modo que se puedan tener ciertos lineamientos a la hora de la escritura. Este ejercicio podría realizarse con estudiantes de todos los grados, pues la dificultad va aumentando a medida que el escritor se va acercando al texto final.

En el ámbito de una escritura académica de artículos, el libro de Zachary Seech (2008) presenta consejos prácticos a la hora de escribir, pues para él, la escritura es la defensa de un planteamiento propio de la historia de la filosofía. Si el tema es claro, el problema de la escritura consiste en que muchas veces se le asigna al estudiante una tarea que no es clara para él. Además, el autor brinda varios criterios a partir de los cuales se puede evaluar el escrito final. Muestra la estructura de un artículo, varios tipos de artículos con los consejos necesarios para hacer un buen trabajo, consejos para comenzar, para revisarlo y para crear un estilo propio. Finalmente, el autor da pautas para citar correctamente. Cabe resaltar que en los apéndices encontramos ejercicios interesantes de edición que pueden utilizarse con estudiantes de todos los cursos.

En Francia, el género académico no es el ensayo como lo hemos visto en los párrafos anteriores, sino el comentario de texto y la disertación. El primero busca fomentar una noción de filosofía como lectura crítica, es decir, el diálogo con el autor en el que "se considere la función que el extracto juega dentro de la obra" (Choulet, Folscheid y Wunenburger, 2011, p. 45). Para ello se deben identificar: el tema, la tesis, la problemática del texto y todas las referencias usadas. Luego se organiza la información según el orden del texto. Aquí se busca que el estudiante no solamente identifique el argumento, sino que logre discriminar la información irrelevante.

La disertación tiene un origen diferente pues fue una forma impuesta en el siglo xIx por el gobierno francés, con el fin de dar una "respuesta fundamentada a una respuesta comprendida" (Gómez, 2003, p. 45). La disertación es, entonces, la exposición organizada del pensamiento a partir de un tema dado (Choulet, et al, 2011). El objetivo de este género está subordinado al tipo de tema que se aborde. De este modo, los cuatro tipos de temas dan lugar a cuatro objetivos diferentes: el primero es una palabra muy general que se usa para generar en los estudiantes una actitud de problematización. La segunda posibilidad es la unión de varios conceptos y se busca trabajar las definiciones antes que la problematización. Un tercer tipo es la pregunta que remite a un problema filosófico y que exige una argumentación clara. Finalmente, se puede trabajar también a partir de una cita con el fin de ver si el estudiante es capaz de tomar una postura personal frente a ella. 
La disertación tiene una forma fija, a saber, un tema, como se mencionó en el párrafo anterior, la tesis o qué se propone del tema, la problemática, el plan y lo que está en juego al sostener una postura u otra. Al igual que el ensayo, la disertación exige claridad y rigurosidad, pero sobre todo exige la problematización o la explicación de por qué el tema de la tesis merece ser discutido filosóficamente. A partir de este problema se determina el modo para solucionarlo, preferiblemente en tres partes, que corresponde al plan. Este debe estar finamente detallado y el paso de un momento a otro debe ser claro. Finalmente, es necesaria la explicación de qué está en juego al sostener una postura u otra para darle mayor fuerza a los argumentos.

\section{Estrategias para lograr la escritura académica}

En el momento en que pensamos la escritura como el fin de un ejercicio pedagógico, llegar a ella se vuelve un reto, incluso en la universidad. El reto en este ámbito consiste en presentar adecuadamente las discusiones filosóficas, sin dejar de lado competencias de lectura y escritura. En este contexto, Malone-France (2008) sugiere que se debe generar un balance entre los contenidos que se enseñan y la enseñanza de la escritura como una habilidad adicional que los filósofos deberían adquirir, pues si bien la historia de las ideas es un reto a pensar diferente, la educación también debería fomentar el desarrollo de la creatividad. En este sentido, el autor propone tres ejercicios. El primero consiste en escribir un ensayo crítico acerca de una serie de textos; el segundo parte del primero y al responderle al autor, se busca que el estudiante desarrolle su propio punto de vista; finalmente, los estudiantes trabajan juntos para corregirse mutuamente y aportar algo nuevo al texto.

Coe (2011) en su artículo Scaffolded writing as a tool for critical thinking: teaching beginning students how to write muestra la importancia de comenzar con ejercicios que lleven a los estudiantes a apropiarse de los problemas y argumentos filosóficos, pues en este ejercicio filosófico encontramos la libertad. De este modo, la autora parte de cuatro ejercicios sencillos: resumir, analizar, comparar y evaluar. El primero genera en los estudiantes discernimiento para saber qué nivel del texto es más relevante. Respecto al segundo ejercicio, es el momento en que los estudiantes logran dar cuenta del rol de un argumento dentro del objetivo general que el autor se plantea y juzgar si es el mejor camino para llegar allí. Para desarrollar la habilidad de comparar, la autora propone una pregunta en común para dos textos filosóficos con visiones opuestas y los estudiantes deben demostrar qué tienen en común o en qué se diferencian. En un cuarto y último momento, se busca que los estudiantes den sus propias definiciones.

Mulnix y Wilson-Mulnix (2010) también presentan estrategias para escribir los ensayos en un contexto universitario. El ejercicio se hace en la forma de un portafolio repartido en nueve momentos a partir de los cuales se llega al ensayo final. El primero es la expresión de un problema bajo la forma de una opinión. En el segundo momento a dicha opinión se le dan argumentos y se organizan en un diagrama de flujo. En tercera instancia se escribe el primer borrador. En el cuarto momento se hace un ejercicio de evaluación por parte de un compañero en el que se deben identificar los argumentos y dar un comentario positivo. Esto permite, en un quinto momento, definir de nuevo el argumento y encontrar una posible objeción. En un sexto momento se hace la respectiva búsqueda bibliográfica con el debido trabajo de fuentes. En un séptimo momento se vuelve a escribir el ensayo con las fuentes adecuadas. Una vez terminado todo, se pasa a un octavo momento de corrección entre los mismos estudiantes y, por último, se entrega una versión final del ensayo.

En el libro Philosophical writing: an introduction (Martinich, 2005) se encuentran excelentes herramientas para escribir un ensayo académico en un contexto universitario. La intención del libro es ayudar a los estudiantes a construir los ensayos y a argumentar mejor, pues la noción de filosofía que este libro maneja parte de la claridad del pensamiento que se traduce en la claridad y rigurosidad de la expresión escrita. De este modo, la filosofía se entiende como el buen uso de la gramática al servicio del análisis de una cierta postura. No en vano el libro presenta ejemplos y ejercicios que involucran la lógica, pues un argumento desorganizado o débil ni es fácil de comprender ni es evidente. Así, encontramos una explicación de las estructuras de los argumentos y qué leyes permiten que estos sean válidos. Entre las herramientas que la lógica puede brindarnos a la hora de escribir encontramos una sección 
sobre las paradojas, contradicciones, los contrarios y la fuerza de las preposiciones. Además, el libro trae unos ejercicios de escritura que acompañan cada reflexión.

Si bien, la lógica es una herramienta fundamental a la hora de escribir, es necesario que el contenido sea verdadero (Martinich, 2005). Son numerosas las dificultades que surgen de esta tesis, pero el autor sugiere que recurrir a la autoridad, a las pruebas científicas puede ser útil siempre y cuando se manejen críticamente; una vez claro el contenido, es posible exponerlo del modo más lógico posible. El libro muestra las dificultades a la hora de definir la forma del ensayo, pues para lograr la complejidad deseada, es necesario ejercitarse en la coherencia, claridad, consistencia y rigor. Gracias a estos elementos, es posible volver sobre la introducción para saber qué evitar a la hora de realizarla. Cabe resaltar, además, que los apéndices al final son guías tanto para el alumno como para el maestro, pues tiene consejos para escribir un ensayo bajo presión, para marcar referencias e incluso para evaluar.

Siguiendo la línea de la escritura académica en los cursos introductorios a la filosofía en la universidad, Joel Feinberg (2013) piensa el quehacer de la filosofía desde la escritura, como el momento de apropiación de las preguntas filosóficas. Una vez más encontramos la precisión gramatical como el elemento básico del que parte la filosofía, aunque no se queda allí, pues el autor aporta elementos estilísticos de gran utilidad como el uso de metáforas, el buen uso de conectores para manejar la cadencia del texto, el cuidado con el uso repetido de algunas palabras preferidas y, finalmente, cuidarse de un estilo opaco o duro de leer. Además, el libro aporta excelentes herramientas lógicas para darle forma a los argumentos. Uno de los grandes aportes que podemos encontrar acá son los enlaces para encontrar bibliografía filosófica en línea y la lista de elementos que el ensayo debe tener.

Dadas las dificultades de la escritura filosófica, el libro Doing philosophy: a practical guide for students (Saunders, Mossley, MacDonald y Lamb, 2008) presenta una serie de consejos sobre lectura, estudio y escritura filosófica en un ambiente universitario. Para los autores, a la filosofía conciernen preguntas por las condiciones de posibilidad del conocimiento, de la realidad y de la acción. De este modo, las técnicas de escritura giran en torno a la producción de ensayos y a todo lo que ello comprende como el manejo del tiempo, escoger la pregunta, estructurar la respuesta, evitar la copia, usar correctamente los recursos y citar en forma apropiada. Este libro, rico en consejos, presenta una guía útil, fácil de leer y muy completa tanto para estudiantes como para maestros que quieren realizar actividades de escritura en clase.

El taller de la filosofía de Nubiola (2002) nos introduce a una escritura que se forja en el amor al pensamiento. A partir de una noción clásica de la filosofía como amor a la sabiduría, la escritura es aquella que nos permite volver sobre nosotros mismos con el fin de aclarar la propia vida. No en vano el autor comienza con las cartas y géneros autobiográficos propios de la filosofía como el diario, pues estos géneros parten de descripciones de un problema y, poco a poco, van desenterrando los sentimientos que han dado lugar a ese problema específico. Esta intimidad cobra un carácter filosófico en el momento en que la ponemos al servicio de la verdad, porque genera compromiso y porque se traduce en la humildad de saber que no todo puede saberse.

Además de destacar la agradable redacción de Nubiola, hay que resaltar que entre los géneros de escritura en filosofía el autor incluye los artículos y la tesis doctoral. Respecto a estos géneros, claramente académicos, el autor sugiere una disciplina férrea para establecer horarios de escritura, esto es, tener la escritura como oficio, porque escribir bien es producto de varios borradores y correcciones. Esta disciplina se hace necesaria a la hora de escribir una tesis, pues por su extensión puede que el autor se pierda en nimiedades o que no se atreva a realizarla. Para ello, es necesario planificar bien el problema y el tiempo que va a requerir.

El texto del profesor Miguel Ángel Pérez Lógica clásica y argumentación cotidiana (2006) nos presenta la lógica como una herramienta que permite organizar el pensamiento y por ello la escritura. Si bien estos ejercicios sirven en cualquier contexto, como un ejercicio de autorreflexión, están incluidos en este apartado porque la argumentación busca apoyar procesos de diferentes disciplinas e incluso de investigación. Así, en los primeros 
capítulos el autor revisa las estructuras de los argumentos y de las razones para luego proponer ejercicios didácticos. Hay un gran aporte en los apéndices, pues allí encontramos las herramientas necesarias para la producción de textos como un examen de los propios prejuicios, y una buena preparación para el texto. Al igual que Nubiola, el profesor colombiano resalta la importancia de una citación adecuada y aporta las herramientas para citar correctamente.

En estos talleres se ha hecho mucho énfasis en la construcción de argumentos y la importancia de seguir las leyes de la lógica, dentro del contexto de la escritura. Cabe resaltar este aspecto, pues el hecho de que los estudiantes conozcan los diferentes tipos de argumentos no garantiza que los sepan usar (Faulconer, Williams y Packard, 1988) Con el fin de resolver este problema, los autores proponen un curso de pensamiento crítico basado en escritura. El énfasis es en gramática, lógica y retórica; las estrategias utilizadas, aunque varían un poco, parten de ejercicios que van aumentando su dificultad y que se realizan siempre en clase. Dichos ejercicios consisten en: corregir la gramática de un párrafo, examinar un argumento o buscar un mejor estilo para la frase. Además, el texto cuenta con unos ejercicios finales muy útiles para realizar en clase.

Los talleres que hemos mencionado hasta ahora parten de la argumentación, dada la visión de la filosofía como defensa de una postura. Los ejercicios de Weiss (2002) también se dan en el contexto universitario, desde la dificultad que los estudiantes tienen a la hora de comprender las ideas filosóficas. El problema radica en que los ejemplos de la historia de la filosofía pertenecen al contexto del filósofo, que muchas veces está alejado de la vida de los estudiantes. En ese sentido, la propuesta de Weiss consiste en usar la escritura para enseñar la historia de las ideas. Podría objetarse que los profesores también ponen ejemplos de su vida cotidiana en clase, pero no hay modo de saber si los estudiantes pueden relacionarse con estos ejemplos. La estrategia de Weiss es tomar la perspectiva de un autor de la filosofía y describir un hecho de la vida real desde allí. Estos ejercicios han tenido un impacto positivo en los estudiantes, pues los ha llevado a ver más claramente el mundo, los textos, un aspecto sobre sus propias vidas y su propia perspectiva sobre las ideas políticas.
Entre los talleres y ejercicios de escritura académica y la escritura a partir de problemas, encontramos la propuesta de Ariane Filius (2016) en la Universidad de Münster, Alemania, quien, a partir de la didáctica de la filosofía, busca dar apoyo a los estudiantes en cualquier forma de escritura en la que ellos estimen que tienen problemas: ensayos, tesis o cualquier otro género académico. En este caso, se busca sobre todo que los estudiantes mejoren la redacción, pues como se ha insistido anteriormente, la claridad y la buena gramática son el fundamento de toda escritura filosófica. La escritura es aquí un medio privilegiado para que el estudiante pueda comprender mejor el texto y organizar sus ideas.

El trabajo de Filius está, además, apoyado por las plataformas web en las que se pueden descargar tutoriales y bibliografía como apoyo a la investigación científica y filosófica. Además, ella promueve una serie de workshops o talleres de escritura filosófica que parten de inquietudes o problemas clásicos de la filosofía y en los que los estudiantes deben dar respuesta a la pregunta. En la medida en que son talleres con una temática bastante amplia, no hay un género impuesto en el que se debe escribir. Esto permite que los estudiantes puedan explorar las inquietudes que tienen y que la escritura se convierta en un espacio creativo. Con esto se trabajan las dificultades de escritura cuando los estudiantes se enfrentan a los géneros académicos, a saber, argumentación, claridad, coherencia, manejo adecuado del problema y desarrollo del estilo.

Dentro de este tipo de propuestas se encuentra el trabajo de Lina Trigos (2016), quien a partir de preguntas personales a los estudiantes los invita a encontrar el valor de la escritura y la argumentación. Ella parte, entonces, no solamente de la propuesta de una tesis, sino además de ejemplos personales que la acercan a los estudiantes. Una vez cada cual ha escogido qué quiere defender, se hace un ejercicio en el que se identifican argumentos y aspectos tanto positivos como negativos. Este ejercicio es útil, puesto que la escritura es un momento para ponerse en los zapatos del otro. Finalmente, la autora recomienda el uso de algunos aspectos retóricos con el fin de que el lector encuentre mayor gusto. 


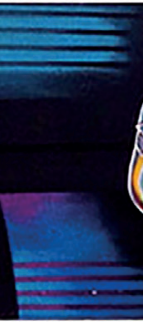

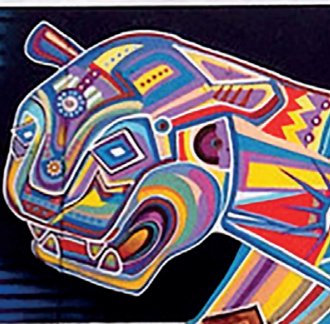

-

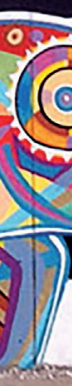

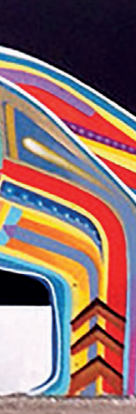
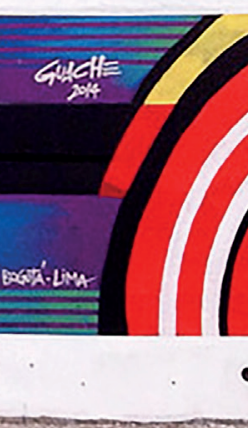
Pensar que si el ejercicio pedagógico se centra solamente en la argumentación pierde su relación con la vida, es dejar de lado iniciativas como la de Laura Duhan (2006). En su clase de introducción a la filosofía ella empieza con ejercicios de lectura, de debate y de escritura de reflexiones. A partir de un texto, la profesora les hace preguntas personales a los estudiantes y les pide que cuenten en qué momentos ellos se han sentido o han pensado como el autor. Esta estrategia permite que los estudiantes piensen en primera persona, entiendan los textos y que a partir de ellos entiendan sus propias vidas. Además, la autora también utiliza un diario de experiencias en el que los estudiantes deben consignar los momentos que los hayan impactado, momentos a partir de los cuales luego se debe escribir un ensayo, una pieza de escritura narrativa. En la medida en que este tipo de ejercicios busca una visión más íntima y privada de la filosofía, Duhan sugiere que al final del semestre se discuta sobre las diferentes formas de hacer filosofía.

Este tipo de talleres son sumamente útiles a la hora de desarrollar la claridad en el lenguaje y en el pensamiento. Esto cobra una mayor relevancia si entendemos que el paso de la opinión y de la comprensión de lectura a un análisis crítico y una argumentación correcta son procesos que se dan en el análisis de diversos géneros de escritura. De este modo, para Walschots (2015), la búsqueda de claridad responde a un ejercicio constante en el que los participantes discuten y evalúan las falencias de otros. Así, aunque la escritura siempre guarde el carácter íntimo propio de expresar los intereses del escritor, ella sigue siendo herramienta de comunicación.

\section{La escritura filosófica como apropiación del conocimiento}

En los talleres anteriores vimos prácticas pedagógicas de escritura que incentivan la argumentación. El ensayo es el género dominante en este caso, pues exige una estructura clara y bien hilada. Este género, propio en la academia, tiene una forma que permite una comprensión rápida del problema y una evaluación clara de los argumentos (Stewart, 2013). En Francia sucede lo mismo con el género de la disertación que, como ya vimos, busca la apropiación de un problema. Estas formas han cobrado una gran relevancia a la hora de definir y de enseñar la filosofía, pues se asume que la forma de escritura permitirá que los estudiantes se apropien de las ideas y sean capaces de pensar por sí mismos.

Respecto al género de la disertación y al comentario de texto, algunos profesores han cuestionado sus presupuestos y sus implicaciones a la hora de enseñar la filosofía. Por una parte, la existencia de varios géneros de escritura en filosofía nos permite ver que no hay algo así como un género privilegiado. Por otra parte, como lo indica Meirieu (citado en Gómez, 2015), dominar las técnicas de una forma o de un género no es suficiente para generar una actividad personal de pensamiento crítico, pues existen personas capaces de seguir las reglas sin generar algún tipo de pensamiento propio. También Onfray (citado en Gómez, 2015) declara que este género solo permite educar a una élite y hacer sufrir a los profesores. De este modo, las apuestas que veremos a continuación son una respuesta en la que la libertad de escribir según el género deseado remplaza el rigor académico con el fin de buscar la apropiación de sí mismo en la escritura.

La apuesta pedagógica de algunos filósofos parte de los diversos géneros literarios en filosofía como la forma de sacar a esta última de la academia y enseñarla de un modo distinto en colegios u otros espacios. En este sentido, hay que destacar el trabajo de Oscar Brenifier (2005) caracterizado por buscar que el alumno desarrolle las competencias necesarias para argumentar y problematizar a partir de la lectura, el debate en clase y el cuento como actividades privilegiadas en las que el estudiante logra una expresión genuina de sí mismo. Para ello, el autor comienza con una exposición sobre el modo como él entiende el quehacer filosófico y la práctica pedagógica con el fin de mostrarnos las ventajas del debate. Decimos que estos talleres parten de la problematización porque el debate debe servir para "articular un problema que ya existe, articulación que no implica necesariamente que el problema se tenga que resolver, sino solo que por lo menos pueda ser abordado." (p. 15). En otras palabras, se trata de ver por qué el problema podría ser examinado, o por qué ese tema amerita nuestra atención y esfuerzo. 
Para aprender a escribir es necesario aprender a leer con detenimiento, a identificar la forma y los argumentos dentro del texto. Esta es tal vez una de las dificultades más grandes que tienen los estudiantes a la hora de aprender por qué las lecturas filosóficas son complejas. De este modo, un primer ejercicio que se puede hacer a partir de la escritura es el de identificar las frases más complejas y explicar por qué no se entiende la lectura. Una vez aclaradas las dudas y entendidos el problema y la hipótesis del autor, el alumno puede escribir su propia opinión acerca de la problemática. Para realizarlo, cada estudiante deberá escribir un pequeño resumen del texto con dos argumentos que soporten la idea principal. Al finalizar, cada uno deberá escribir una pregunta o una objeción a la hipótesis. El objetivo de esta actividad consiste en que los estudiantes puedan proponer una visión propia del problema y la puedan argumentar.

Brenifier (2005) además propone un ejercicio para rescatar el estatuto del ejemplo en filosofía. La idea principal es contar muchos cuentos que aborden una problemática y al final se escoge el cuento que mejor la aborde. Luego se analiza la historia de acuerdo al modo como se dio cuenta del problema y se cuestiona, bien sea a partir de una nueva hipótesis o con diferentes preguntas. Esta confrontación de las hipótesis es importante porque muchas veces una cierta posición puede acarrear graves consecuencias, así que es necesario examinar qué está en juego cuando afirmamos algo. Así, el ejercicio final consiste en volver a elaborar el cuento si es necesario. En párrafos anteriores vimos tan solo algunos ejemplos de los ejercicios que pueden llevarse a cabo, sin embargo, Brenifier y Millon (2014) han desarrollado cuentos y estrategias útiles para dar a conocer las preguntas filosóficas en contextos no académicos.

También en Francia los talleres de Michel Tozzi (2000) han cobrado una gran importancia pues proporcionan un espacio interesante para pensar problemas filosóficos por fuera de la academia, pero sobre todo por fuera del género de la disertación. En este sentido, el profesor francés retoma la escritura como un diálogo del autor consigo mismo (Tozzi, 2012). Así, los géneros literarios en filosofía como los aforismos, los cuentos, los juegos de palabras, etc., sirven para buscar nuevas intuiciones, para pensar un problema o formular mejor aquellas ideas que ya se venían trayendo. Se ha demostrado que los participantes no solamente aprecian los ejercicios y asisten con regularidad, sino que logran alcanzar los objetivos planteados.

Estos ejercicios son considerados filosóficos porque manejan tres competencias básicas en filosofía: la problematización, la argumentación y la conceptualización. La primera consiste en cuestionar la pregunta y cuestionar sus presupuestos con el fin de mostrar por qué una cierta hipótesis merece ser evaluada o qué puede seguirse de adoptar una cierta visión u otra. Una vez tenemos un problema, hay que decir por qué se adopta una cierta visión o una cierta tesis. Para ello la argumentación clara, concisa y organizada es necesaria. Finalmente, estos argumentos que dan cuenta del problema nos permiten crear nuestros propios conceptos.

La diferencia entre lo que hace Tozzi y la disertación consiste en que el profesor francés usa los aforismos, las cartas y los debates para llegar a los argumentos (Tozzi, 2007). La metodología consiste en partir de un tema, analizarlo y proponer una idea. Luego se debate al respecto, con el fin de generar nuevos puntos de vista. El papel del filósofo es ser un animador de la discusión, es decir, dar la palabra, sintetizar las ideas y evitar que solo un integrante se lleve la palabra. Una vez hecho esto, se puede pasar a la escritura individual como un momento de reflexión íntima a partir de lo que acaba de pasar. Esto permite un espacio diferente que es la lectura de textos en voz alta y finalmente la escritura de un aforismo.

Los debates tienen una gran importancia en el ejercicio de la filosofía, pues es el momento en que el estudiante se atreve a discutir su interpretación. Este ejercicio se usa para que el profesor no sea el único que tome la palabra. Para Roberts (2000) esta práctica permite saber si los estudiantes han entendido las ideas generales de un texto. Para saber si se han comprendido los detalles específicos, se les pide a los estudiantes que escriban un párrafo corto al final de la clase. Otra estrategia que ayuda a evaluar la comprensión es que los estudiantes se corrijan mutuamente, pues es necesario tener conocimiento del tema para evaluar a otro. Para el autor estos métodos funcionan porque garantizan que se escriba constantemente en los cursos y que se genere una familiaridad con la escritura que permite una corrección ágil durante las tutorías. 
En la Universidad popular de filosofía en Toulouse se llevan a cabo talleres de escritura todos los martes a cargo de Geneviève Bonifait (2015). Durante tres horas, los participantes deberán crear un texto a partir de la imaginación y de las habilidades de escritura que ya tengan. Para ello, bien puede partirse de una pregunta filosófica o de una lectura breve. En este caso, la animadora sugiere que los textos despierten las emociones de las personas. Finalmente, los textos se leen en voz en alta o los unos a los otros. Esto exige siempre un buen comportamiento y respeto por parte de los participantes que se asegura a partir de una buena disposición.

Como parte de un proyecto de hacer de la filosofía un ejercicio para todo aquel que esté interesado en ella, en Biarritz, Francia, existen talleres de filosofía animados por Christophe Lamoure (2011). Las actividades son muy variadas, pues tienen clubes de lectura, seminarios y además un taller de escritura filosófica en el que los asistentes deben desarrollar la claridad, el rigor y la capacidad de dialogar, que son las competencias básicas de la filosofía. Dado el interés por fomentar la lectura, el animador parte de textos filosóficos cortos como el Enquiridión de Epicteto. Los estudiantes deben dialogar con los textos de manera escrita y con la libertad de escoger el género deseado. Entre los ejemplos que el autor aporta sobre escritura filosófica encontramos una receta de cocina, reflexiones, textos con la misma forma del autor, etc. Estos ejercicios, más allá de ser agradables, buscan generar una apropiación del conocimiento a partir de la escritura.

El uso de los textos filosóficos es bastante controversial en los talleres, pues, para Isabelle Pariente (2012) estos pueden ser un obstáculo para el escritor. En filosofía hay un rigor que muchas veces puede confundirlo o hacerle tomar una posición que no le es propia. Un texto no es filosófico por su análisis de un autor propio de la historia de la filosofía, sino por la capacidad que tiene de transformarse a sí mismo en el pensamiento. Así, ella propone ejercicios que parten de frases y que el escritor debe desarrollar en el texto.

De la misma manera, a partir de un problema que se piensa primero en la intimidad y luego en el diálogo con el otro, Mathilde Vermer (2015) anima unos talleres de escritura con el fin de que los asistentes piensen su realidad. De nuevo, encontramos una noción de filosofía como aquello que nos permite asir el mundo a partir de conceptos propios y que permite expresar nuestra diferencia. En esta misma línea de pensamiento está Jean Paul Gilbert (2016) que propone en su sitio web una serie de preguntas abiertas o de frases que sirven de comienzo para que cada participante conteste. Lo interesante de ambos ejercicios es que, al partir de una frase ambigua, los participantes pueden explorar los géneros y las ideas que crean conveniente y al final obtendrán una retroalimentación doble de los otros participantes y de sí mismos.

A partir de estas propuestas, Miguel Ángel Rosa (2011) ha desarrollado propuestas de talleres de escritura dentro del aula de clase no tanto porque sea un modo más agradable de enseñar, pero sí porque permite una apropiación del conocimiento. Así, él parte de un problema y entre todos los estudiantes deben determinar por qué eso es un problema y qué argumentos se dan a favor. Este proceso que se acaba de mencionar es la preescritura. Luego, los estudiantes se leen unos a otros y se reescribe el texto según los comentarios. Otro modo de realizar esta actividad es con fichas bibliográficas que los estudiantes deben usar para construir el texto. Otro ejercicio posible consiste en dividir a la clase en varios grupos, tomar un problema filosófico y pedirle a cada grupo que realice una actividad diferente respecto al texto. Después cada grupo expone y, finalmente, se escribe a favor o en contra de los argumentos. Si bien puede llegarse a la escritura de géneros académicos, el fin del profesor Rosa consiste justamente en partir del gusto de escribir, aunque sus clases no se limitan a eso, porque lo importante es entender el lugar que el conocimiento juega a la hora de forjar una vida mejor.

En todos los talleres anteriores había una clara exigencia en la escritura y en el planteamiento de un pensamiento propio, libre y crítico. A diferencia de los primeros talleres, en este segundo apartado vimos un camino diferente para llegar a dicha forma de pensamiento, a saber, a partir del diálogo como herramienta para buscar claridad. En este sentido, el uso de la imaginación y de la creatividad devienen medios de expresión de sí mismo y de apropiación vital del conocimiento. Cabe resaltar que estos talleres se hacen fuera del espacio académico, no porque sea un modo ameno de enseñar, sino porque 
ni la evaluación ni la publicación son los fines de dichas tareas. El reto consiste en motivar a los participantes a explorar sus propias ideas y a hacerles ver la relación entre el conocimiento y la expresión de sí.

\section{La escritura como herramienta para el desarrollo del pensamiento crítico}

En los apartados anteriores hemos visto la importancia del pensamiento crítico y el papel de la escritura y del diálogo en el desarrollo de este proceso. Ahora bien, llegar a escribir o pensar de modo argumentado es un proceso que muchos rehúyen, porque jamás desarrollaron las capacidades para pensar ni escribir así. De este modo, es necesario desarrollar estrategias que ayuden a los estudiantes a responder con asertividad y a perder el miedo al papel en blanco, al no saber qué decir. Con estos ejercicios se busca eliminar este temor, y se procura hacer de la escritura un proceso que comienza con borradores, como lo ha pensado Fishman (1989). En la experiencia de este profesor, este tipo de ejercicios pueden ser útiles cuando están unidos a un ensayo o cuando son realizados continuamente. En lo que veremos a continuación, hay una preocupación por generar las competencias necesarias para desarrollar la escritura como proceso con miras a generar pensamiento crítico.

Dado que muchas veces los problemas filosóficos parecen demasiado abstractos, existen métodos para que los estudiantes se introduzcan a la filosofía a partir de otras actividades como el cine, los cuentos, la creación de revistas y el teatro. En Didáctica de la filosofía, Víctor Santiuste y Francisco Gómez de Velasco (1984) exponen un taller de filosofía basado en las experiencias de los estudiantes, pues se ha demostrado que los jóvenes "entran en este universo mejor a través de la acción que de la palabra" (p. 143). En este sentido, se recomienda comenzar con preguntas, debates y escritos que no se someten a una sola forma, sino que exploran los gustos del estudiante y que terminan por forjar una actitud crítica ante el entorno.

Esta corriente de talleres que expondremos busca incentivar la filosofía desde la escritura creativa, aunque, a diferencia de los talleres planteados en la sección anterior, el objetivo es poder desarrollar las herramientas y habilidades que los estudiantes necesitan para comprender la abstracción de la filosofía. Teniendo esto en cuenta, estos talleres servirían para preparar a los estudiantes para los ejercicios vistos en la primera categoría. En ese sentido, el trabajo de Matthew Lipman (2003) se destaca por hacer de los problemas filosóficos un punto de partida de debates, y de escritura, con el fin de generar en los estudiantes el orden, la expresión, la creatividad y el análisis necesarios para obtener una actitud crítica. Para ello se parte de una historia, normalmente contextualizada en el colegio. Lo interesante de las historias es que cada una de ellas presenta diferentes problemas filosóficos. Por ejemplo, Elisa (Lipman, 2003) trabaja temas de ética como comer animales, las mentiras, la amistad, etc. Marcos (Lipman, 2004) es un libro en el que encontramos mayor énfasis en problemas de la adolescencia como el hecho de crecer en medio de una sociedad con valores que muchas veces no comprendemos o no queremos aceptar. Kio y Gus gira en torno a la ciencia y a las competencias que se requieren para razonar correctamente.

El trabajo de Lipman cuenta, además, con un manual para el profesor en el que se sugieren ejercicios de lectura, de debate, de escritura y de proyectos. Cada libro cuenta con su propio manual, aunque aquí destacaremos el del libro Suki, pues su objetivo es generar en los estudiantes el paso de los debates y la lectura a la escritura. Escribir: cómo y por qué (Lipman, 2000) parte de la idea de que el pensamiento filosófico exige un orden que solo puede darse en la escritura, pues la escritura exige discernimiento, decisiones conceptuales y producción lógica, sin importar el género. Además, la escritura exige ritmo. El objetivo del libro consiste en poder transitar del discurso a la escritura y viceversa. Para ello, el autor propone una serie de ejercicios basados en la poesía que desarrollan en el estudiante la habilidad de comprender diferentes significados para una misma palabra, la abstracción de reglas a partir de ejemplos, la habilidad para retomar el texto cuando esté bloqueado y, en general, para que gracias a la comprensión de las infinitas posibilidades del lenguaje, el estudiante se exprese poética y académicamente.

El papel de la escritura como herramienta que ayuda a desarrollar el pensamiento también puede verse en libros que, aunque no se preguntan qué es la escritura, 
la usan como herramienta para el desarrollo del pensamiento crítico. Por una parte, Marcos (Lipman y Sharp, 1990), que versa sobre los problemas sociales, cuenta con el comentario filosófico como una herramienta para aprender a leer críticamente y poder proponer de modo escrito algo respecto a la lectura. Por otra parte, en Kio y Gus (Lipman, 1993), se busca desarrollar las habilidades mentales necesarias para la ciencia, y para ello se usa la escritura porque esta permite desarrollar habilidades de lectura crítica y relatar una experiencia a partir de la poesía. El objetivo de este último ejercicio consiste en hacer del conocimiento una experiencia de atención y cuidado hacia el objeto investigado. Si bien el trabajo de Lipman ha sido criticado y revaluado (Brenifier, 2003), sigue siendo una referencia fundamental para probar que el gusto por la filosofía y las habilidades que ésta genera pueden desarrollarse desde temprana edad.

Philip Cam (2006) también se encuentra en la línea de Matthew Lipman, pues a partir de cajas de herramientas o metodologías que pueden utilizarse en el salón de clase, se puede aprender a pensar y a examinarse a sí mismo. Estas herramientas son los recursos que encontramos en la filosofía clásica como cuestionar, proponer hipótesis, evaluar, Ilevar un registro, conceptualizar, razonar o preguntarle a la pregunta, dichas herramientas varían según los niveles de los estudiantes. El libro presenta, entonces, estrategias para llegar a la argumentación a partir del desarrollo de habilidades más básicas como: dar ejemplos, distinguir y dar cuenta de los criterios que utilizamos a la hora de tomar una decisión. La escritura es necesaria en el desarrollo de estas habilidades, pues el escribir en el papel los ejercicios mencionados anteriormente permite una reflexión profunda y orden en el pensamiento. Así, la escritura da lugar a una forma de abstracción privilegiada.

Para Robert Fisher (2016), los cuentos, juegos y poemas son un estímulo importante que los estudiantes pueden tomar para empezar a pensar. Su objetivo principal es lograr que los estudiantes cuestionen el escrito y que discutan con sus compañeros sobre la idea principal. Así, además de la diversidad de cuentos que encontramos, el autor da unas pautas para pensar en común, como el análisis del orden temporal, de eventos particulares, de las intenciones, de los significados y del relato mismo. Una vez hecho este análisis profundo, el estudiante será capaz de responder las preguntas que el autor plantea y de llegar a sus propias conclusiones sobre el cuento. Finalmente, se trabaja un escrito en el que el estudiante puede expresar los conceptos que le parezcan pertinentes y las partes del cuento que le hayan Ilamado la atención. Este escrito busca generar creatividad en el estudiante y la habilidad de problematizar, de cuestionar y de discernir cuál es el mejor argumento.

Jeff Mason (1992) también trabaja ejercicios de escritura en el salón de clase que buscan forjar en los estudiantes un pensamiento crítico a partir de habilidades como la flexibilidad intelectual y de la creatividad. Para ello, él comienza con el "précis" o tarea de reducir un texto. Como esto es, a simple vista, imposible, su tarea consiste en entender la idea principal, escoger los argumentos más fuertes y ser fiel a la forma original. Un segundo ejercicio es el de resumir y parafrasear, que se diferencia de lo anterior en la medida en que produce un texto nuevo, pues ya la forma la escoge el escritor. Esto pone en evidencia que muchas veces el argumento depende de su forma y que, al ser extrapolado, el sentido puede cambiar. Finalmente, se sugiere como ejercicio de escritura problematizar y cuestionar el texto desde adentro, es decir, desde lo que el autor plantea como argumento.

La iniciativa de Michael Boylan (2010) es bastante interesante y completa puesto que, con el fin de desarrollar ambos lados del cerebro, el autor retoma los conceptos, cuentos y argumentos propios de la historia de la filosofía con el fin de desarrollar no solamente las competencias necesarias para argumentar, sino además para desarrollar la creatividad. A partir de textos sobre las grandes figuras de la filosofía como Platón, Aristóteles, Budha, Santo Tomás, Descartes, Marx, Kant, Heidegger, Arendt, Luther King, el autor busca, por una parte, que los estudiantes identifiquen los argumentos y comprendan cómo responder desde y a esa forma del discurso. Por otra parte, la escritura de cuentos es un esfuerzo por responder desde las habilidades creativas que estos ejercicios generan.

En estos talleres pudimos ver cómo el problema se pone al servicio de la habilidad que se quiera fortalecer y de 
las respuestas a preguntas que atañen a nuestra vida. Los cuentos están diseñados de manera que los estudiantes desarrollen habilidades tales como: organización de la información, expresión de sí mismos, cuidado de su objeto de estudio, creatividad, flexibilidad, el uso de la lógica y de la posibilidad de dar criterios para responder a un problema. La escritura sirve, entonces, como un medio privilegiado para desarrollar todas las partes del cerebro y para recordarnos que el ejercicio intelectual es un proceso que sí puede forjarse desde la infancia.

\section{Conclusiones}

En las tres formas de talleres pudimos apreciar cómo las diferentes nociones de filosofía y los diferentes públicos generan ciertas metodologías que pueden ser útiles tanto dentro como fuera del salón de clase. Esta categorización es relevante para dar luz a diferentes prácticas que se están llevando a cabo en otras facultades y para pensar nuevas formas de prácticas filosóficas. En la medida en que estos talleres se realizan tanto en universidades y colegios como fuera de ellos, las pautas que aquí encontramos son oportunidades no solo para reflexionar sobre la propia práctica docente, sino para pensar formas de la enseñanza que saquen a la filosofía de la academia.

En este sentido, entrar al debate sobre las diferentes formas de incentivar la escritura filosófica implica reconocer los diferentes estilos, géneros y procesos a partir de los cuales se ha hecho y se hace aún la filosofía. Esto es relevante, pues implica reconocer que, aunque la mayoría de esfuerzos giran en torno a la publicación, esto no es ni el único modo ni el único fin del quehacer filosófico. La filosofía también es producto del diálogo, de los debates y del modo como interpretamos la existencia. Así, la filosofía parte de la vida y nos permite vivir mejor, pues tener las herramientas propias del pensamiento crítico no es simplemente algo útil a la hora de escribir, sino para poder decidir autónomamente qué vida deseamos llevar y qué decisiones nos llevan por ese camino.

A modo de reflexión final, cabe decir que, aunque no estuvo del todo presente una noción de transformación, sí se podría rastrear alguna noción del modo como la escritura puede generar una cierta transformación. $\mathrm{Al}$ atender a lo que los autores proponen, los primeros talleres generan una transformación, pues permiten el paso de un pensamiento oscuro y vacío a una postura bien expresada y bien argumentada. Los segundos talleres permiten una transformación porque a partir de la escritura el conocimiento se vuelve una herramienta para vivir mejor. Finalmente, el tercer tipo de taller genera la transformación propia de quien adquiere nuevas herramientas de pensamiento. Lo dicho anteriormente permite rastrear un beneficio invaluable de la escritura, que al volverse hábito y fomentarse puede Ilevarnos a vivir una mejor vida.

\section{Referencias}

Bonifait, G. (2015). Ateliers d'écriture philosophique. Recuperado de: http://www.alderan-philo.org/content/atelier-décriture

Boylan, M. (2010). An innovative introduction to philosophy: fictive narrative, primary texts and responsive writing. Colorado: Westview Press.

Brenifier, O. (2005). El diálogo en clase. Tenerife: Idea.

Brenifier, O. (2007). La pratique de la philosophie à I'ecole primaire. Paris: Alcofribas Nassier. Recuperado de http://www.pratiquesphilosophiques.fr/wp-

Brenifier, O. y Millon, I. (2014). Cuadernos de ejercicios filosóficos. París: Instituto de prácticas filosóficas. Recuperado de: http:// www.pratiques-philosophiques.fr/wp-content/uploads/2014/01/ Cuaderno-111-ejercicios.pdf

Cam, Ph., (2006). 20 thinking tools. Victoria: Acer Press.

Choulet, Ph, Folscheid, D. y Wunenburger, J-J. (2009). Méthodologie philosophique. Paris: P.U.F.

Chudnoff, E. (2007). Guide to philosophical writing. Massachusetts: Harvard University Press.

Coe, C. (2011). Scaffolded writing as a tool for critical thinking: teaching beginning students how to write arguments. Teaching philosophy, 34, 33-50.

Cruz, J. (2005). Paper writing strategies for introductory courses. Recuperado de: https://web.williams.edu/wp-etc/philosophy/ jcruz/jcruz/writingtutor/index.html

Duhan, L. (2006). Autobiographical writing in philosophy classes. Teaching philosophy, 29, 23-36.

Faulconer, J., Williams, R. y Packard, D. (1988). Use critical reasoning to teach writing. Teaching philosophy, 11, 229-244.

Feinberg, J. (2013). Doing philosophy. California: Wadsworth.

Filius, A. (2016). Schreibenwerkstatt des Philosophichen Seminars. Recuperado de: https://www.uni-muenster.de/PhilSem/mitglieder/schreibwerkstatt/schreibwerkstatt.html 
Fisher, R. (2016). Stories for thinking. Recuperado de: http://w. verbalartscentre.co.uk/T3/assets/Professor\%20Fisher's\%20 Handout.pdf

Fishman, S. (1989). Writing philosophy. Teaching philosophy, 12, 361-374.

Gilbert, J. (2016). Interlude. Recuperado de: https://interludephilo. wordpress.com/

Gómez, M. (2003). Introducción a la didáctica de la filosofía. Pereira: Papiro.

Gómez, M. (2015). Enseñar filosofía: Competencias, disertación, discusión, prácticas, didáctica, saber. Pereira: Universidad Tecnológica de Pereira.

Lamoure, Ch. (2011). Atelier de philosophie Biarritz- Pays Basque. Recuperado de: http://atelier-philosophie-biarritz-paysbasque.e-monsite.com/pages/atelier-d-ecriture.html

Lipman, M. (2000). Suki. Trad. y adaptación Diego Pineda, $1^{\text {a }}$ ed., Bogotá: Editora Beta.

Lipman, M. (2003). El descubrimiento de Harry. Traducción y adaptación Diego Pineda, 2a. ed., Bogotá: Editora Beta.

Lipman, M. (2003). Elisa. Traducción y adaptación Diego Pineda, 2a ed., Bogotá: Editora Beta.

Lipman, M. (2003). Kio y Gus. Traducción y adaptación Diego Pineda, $2^{\text {a }}$ ed., Bogotá: Editora Beta.

Lipman, M. (2003). Pixi. Traducción y adaptación Diego Pineda, 2a ed., Bogotá: Editora Beta.

Lipman, M. (2004). Marcos. Traducción y adaptación Diego Pineda, 1ª ed., Bogotá: Editora Beta.

Lipman, M. y Sharp, A. (1990). Investigación social. Manual del profesor para acompañar a Mark. Madrid: Ediciones de la Torre.

Lipman, M. y Sharp, A. (1993). Asombrándose ante el mundo. Manual del profesor para acompañar a Kio y Gus. Madrid: Ediciones de la Torre.

Lipman, M. y Sharp, A. (2000). Escribir: cómo y por qué. Libro de apoyo para el docente para acompañar a Suki. Buenos Aires: Manantial.

Martinich, A. (2005). Philosophical writing, an introduction. Victoria: Blackwell Publishing.

Malone-France, D. (2008). Composition pedagogy and the philosophy curriculum. Writing philosophy, 1, 59-86.

Mason, J. (1992). The future of thinking, rhetoric and liberal arts teaching. Londres: Routledge.

Nubiola, J. (2002). El taller de la filosofía. Pamplona: Eunsa.
Pariente, I. (2012). Aux bordes des mondes. Recuperado de: http:// auxbordsdesmondes.fr/spip.php?article646

Pérez, M. (2006). Lógica clásica y argumentación cotidiana. Bogotá: Javegraf.

Roberts, R. (2002). Teaching writing-intensive undergraduate philosophy courses. Teaching philosophy, 25, 195-211.

Rosa, M. (2011). La escritura en el aula como práctica filosófica. Revista Digital del Centro de Profesores de Alcalá de Guadaíra, I (9). 1-12.

Santiuste, V. y Gómez de Velasco, F. (1984). Didáctica de la filosofía. Teoría, métodos, programas, evaluación. Madrid: Narcea.

Saunders, C., Mossley, D., MacDonald, G. y Lamb, D. (2008). Doing philosophy: a practical guide for students. Londres: Continuum.

Seech, Z. (2008). Writing philosophy papers. California: Wadsworth.

Solomon, R. (2001). Writing Philosophy En The Big Questions: A Short Introduction to Philosophy, 6th ed. Belmont, California: Wadsworth.

Stewart, J. (2013). Unity of content and form in philosophical writing. Londres: Bloomsbury.

Tozzi, M. (ed.) (2000). Diversifier les formes d'écriture philosophique. Ateliers d'écriture et pratiques de classe. Languedoc-Rouisillon: CRDP.

Tozzi, M. (ed.) (2007). Les ateliers d'écriture philosophique. Recuperado de: http://www.philotozzi.com/2007/05/atelier-dcriturebages/

Tozzi, M. (ed.) (2012). Dictionnaire philosophique. Recuperado de: http://www.philotozzi.com/2012/08/dictionnaire-surlapprentissage-du-philosopher-et-les-nouvelles-pratiquesphilosophiques/

Trigos, L. (2016). Escribir es aprender a pensar. Recuperado de: https://www.uv.mx/noticias/2014/11/10/escribir-es-aprendera-pensar-mejor-lina-trigos/

Vermer, M. (2015). Ateliers d'écriture, les ateliers de Mathilde. Recuperado de: http://www.mathildevermer.fr/ateliers-ecriture/

Walschots, M. (2015). Philosophical Writing: The Essay and Beyond, Teaching Innovation Projects, (58) 1, Article 4. Recuperado de: http://ir.lib.uwo.ca/tips/vol5/iss1/4

Weiss, P. (2002). Making history of ideas classes relevant: A writing strategy. Teaching philosophy, 25, 123-130.

Wilson-Mulnix, J. y Mulnix, M. J. (2010). Using a writing portfolio project to teach critical writing thinking skills. Teaching philosophy, 33, 27-54. 


\section{Diálogo del conocimiento}

\section{La escritura como experiencia de formación}

Conversando con la lectura de "Estado del arte sobre talleres de escritura filosófica"

\section{Conversar con un texto}

Conversar con un texto es siempre conversar en torno y desde la propia lectura del texto. Cuando converso con un texto estoy indagando mi propia lectura del texto, mi propia experiencia de lectura. Y lo hago desde mi propia historia como lector y como sujeto de discurso. Converso con el texto a través del saber sobre los textos que he construido en mi experiencia como lector. No puedo evitar traer mi experiencia a la lectura. Es inconcebible la lectura sin la experiencia de otras lecturas. Conversar por escrito con un texto, o con la lectura de ese texto, es producir un nuevo texto que no podría ser escrito sin la experiencia de la lectura de ese y otros textos. Conversar con un texto es decir o escribir lo que nos da de pensar su lectura.

\section{La escritura como práctica pedagógica}

El texto de Ana Isabel Durán Vélez me da a pensar sobre la escritura como experiencia de formación y los "talleres de escritura" como dispositivos pedagógicos, como estrategias y tácticas de organización, regulación y habilitación de determinadas prácticas discursivas y de producción textual, más o menos institucionalizadas, que trans-forman a quiénes se involucran con ellas. Tal como lo venimos haciendo con los talleres de documentación narrativa de experiencias pedagógicas, la experiencia de escritura puede ser pensada como metáfora de la formación: aprender a escribir textos especializados es el contrapunto y la condición de constituirse como hablante competente de una determinada comunidad de prácticas y discursos. Formarse mediante la escritura es también afiliarse a una comunidad de interpretación. Aprender a escribir textos filosóficos o pedagógicos es aprender una parte importante de los secretos del oficio de la filosofía o de la pedagogía.

Pero toda práctica de escritura y de lectura, toda experiencia de formación, se hace en determinadas condiciones (históricas, institucionales y políticas) de enunciación, están reguladas por reglas de composición más o menos explícitas y son valoradas por criterios de ponderación específicos. Por eso, toda formación, toda escritura, toda lectura y toda conversación están inmersas en las relaciones de poder, de saber y discursos que definen sus límites, alcances y posibilidades, y que configuran y delimitan posiciones, disposiciones y oposiciones de los sujetos (de enunciación, de formación) en ese territorio. Toda práctica discursiva y de formación se despliega y tramita en un campo surcado por diferencias que establecen jerarquías, autorizaciones, subalternidades, y en los que se consolidan o declinan géneros, estilos, recursos y competencias.

\section{Escribir textos en géneros consagrados}

Mi experiencia de lectura del texto me hace pensar en qué es hacer filosofía (o pedagogía). O mejor, me hace preguntar: ¿qué se considera legítimamente como hacer filosofía (o pedagogía)? Para el mundo académico, para las formas consagradas y autorizadas institucionalmente, hacer filosofía (o pedagogía) es escribir ensayos, disertaciones, comentarios de textos filosóficos (o pedagógicos) o tesis. Y formarse en filosofía académica (o en pedagogía académica) es reconocerse y ser reconocido como aquel que es capaz de escribir textos argumentativos, problematizadores y conceptuales, caracterizados por el método, el rigor, la lógica y la gramática, fácilmente reconocibles como parte de una tradición intelectual altamente selectiva, e incorporables al acervo discursivo de las posiciones dominantes del campo. Por eso, para afiliarse a la comunidad de prácticas y discursos consagrados de la filosofía (o de la pedagogía) dominante hay que

Rollos nacionales / Estado del arte sobre talleres de escritura filosófica $\mathbf{5 3}$ Ana Isabel Durán Vélez / 
pasar la prueba de la escritura especializada o técnica, hay que sortear la evaluación de la adecuación y el ajuste del texto producido a las formas institucionalizadas de escribir filosofía (o pedagogía). La escritura profesionalizada de textos filosóficos (o pedagógicos) supone conformarse a sí mismo como un hablante competente de una lengua o, más precisamente, de un juego de lenguaje. Por eso, la escritura especializada de textos (filosóficos o pedagógicos) merece tramitarse a través de dispositivos pedagógicos (los talleres) que, como sabemos, son también dispositivos de poder, saber y discurso.

\section{Otras escrituras, otras experiencias de formación, otras filosofías (y pedagogías)}

Pero más allá de la ingenuidad exhaustiva y de los silencios regulados de un estado del arte escrito como ensayo, la lectura del texto de Ana Isabel Durán Velez también me invita a reconocer e imaginar otras posibilidades y oportunidades de escritura, de formación y de entender y hacer filosofía (y pedagogía). Formas otras que van "más allá de la academia" y de sus géneros y estilos consagrados, de su método riguroso, de sus presiones y restricciones, las cuales se mezclan con la vida y con la experiencia de habitar y decir el mundo y sus territorios de prácticas y discursos como hablante reflexivo, crítico, sagaz. Formas de hacer y escribir la filosofía (y la pedagogía) que la abren a la experiencia de vivir en una determinada geografía y momento histórico, que la localizan en coordenadas que se deslizan más allá de las formas consagradas, que disputan legitimidad y habilitan otras posiciones de sujeto de discurso, poder y saber filosófico (o pedagógico). Estas otras prácticas de escritura, lectura, conversación y formación podrían configurar experiencias disruptivas y rebeldes que conectan las escrituras de sí (narrativas autobiográficas) y de géneros discursivos diversos (cuentos, poesías, aforismos, juegos de palabras, cartas, debates) con la posibilidad de pensar filosóficamente (o pedagógicamente) junto con otros sobre y en el mundo que habitamos, transitamos y luchamos.

Daniel H. Suárez 